\title{
Effect of epithelial debridement on human cornea proteoglycans
}

\author{
E.S. Soriano ${ }^{1}$, \\ M.S.Q. Campos², \\ J.A.K. Aguiar ${ }^{1}$ and \\ Y.M. M ichelacci ${ }^{1}$
}

\author{
Departamentos de ${ }^{1}$ Bioquímica and ${ }^{2} \mathrm{O}$ ftalmologia, \\ Escola Paulista de Medicina, Universidade Federal de São Paulo, \\ São Paulo, SP, Brasil
}

\section{Correspondence \\ Y.M. Michelacci \\ Disciplina de Biologia Molecular \\ Departamento de Bioquímica \\ EPM, UNIFESP \\ Rua Três de Maio, 100 \\ 04023-020 São Paulo, SP \\ Brasil \\ Fax: +55-11-5573-6407 \\ E-mail: yara.bioq@epm.br \\ Presented at the 6th Brazilian \\ Symposium on Extracellular \\ Matrix - SIMEC, Angra dos Reis, \\ RJ, Brazil, September 24-27, 2000. \\ Research supported by CNPq, CAPES and FAPESP.}

Received October 19, 2000 Accepted February 5, 2001

\section{Abstract}

Corneal transparency is attributed to the regular spacing and diameter of collagen fibrils, and proteoglycans may play a role in fibrillogenesis and matrix assembly. Corneal scar tissue is opaque and this opacity is explained by decreased ultrastructural order that may be related to proteoglycan composition. Thus, the objectives of the present study were to characterize the proteoglycans synthesized by human corneal explants and to investigate the effect of mechanical epithelial debridement. Human corneas unsuitable for transplants were immersed in F12 culture medium and maintained under tissue culture conditions. The proteoglycans synthesized in $24 \mathrm{~h}$ were labeled metabolically by the addition of ${ }^{35} \mathrm{~S}$-sulfate to the medium. These compounds were extracted by $4 \mathrm{M} \mathrm{GuHCl}$ and identified by a combination of agarose gel electrophoresis, enzymatic degradation with protease and mucopolysaccharidases, and immunoblotting. Decorin was identified as the main dermatan sulfate proteoglycan and keratan sulfate proteoglycans were also prominent components. When the glycosaminoglycan side chains were analyzed, only keratan sulfate and dermatan sulfate were detected ( $\sim 50 \%$ each). Nevertheless, when these compounds were ${ }^{35} \mathrm{~S}-$ labeled metabolically, the label in dermatan sulfate was greater than in keratan sulfate, suggesting a lower synthesis rate for keratan sulfate. ${ }^{35} \mathrm{~S}$-Heparan sulfate also appeared. The removal of the epithelial layer caused a decrease in heparan sulfate labeling and induced the synthesis of dermatan sulfate by the stroma. The increased deposit of dermatan sulfate proteoglycans in the stroma suggests a functional relationship between epithelium and stroma that could be related to the corneal opacity that may appear after epithelial cell debridement.

The cornea consists of a thick central portion, the stroma, that constitutes about $90 \%$ of the corneal thickness, lined anteriorly by an epithelial cell layer and posteriorly by an endothelium. The two cell layers are separated from the stroma by basement membranes. The cornea is characterized by its transparency and curvature, which provide the initial focusing of the light image

\section{Key words}

- Corneal explants

- De-epithelization

- Glycosaminoglycan

- Proteoglycan into the eye. The stromal cells secrete an extracellular matrix composed mainly of collagen fibrils surrounded by proteoglycans, arranged in orthogonal lamellae (1).

Proteoglycans are macromolecules composed by a protein core with covalently linked glycosaminoglycan side chains (2). In many species, the predominant corneal glycosaminoglycans are dermatan sulfate and keratan 
sulfate, with smaller amounts of heparan sulfate. In 1992, a cDNA clone encoding the core protein of a chick corneal keratan sulfate proteoglycan, named lumican, was obtained (3), and the bovine (4) and human (5) lumican core proteins were later cloned. Two other keratan sulfate proteoglycans, named keratocan and mimecan (or osteoglycin), were cloned from the bovine cornea (6). It seems that although expressed in other tissues, only in the cornea are lumican, keratocan, and mimecan glycosylated with sulfated keratan sulfate chains. The main chick corneal dermatan sulfate proteoglycan was identified as decorin (7).

The transparency of the cornea is attributed to the regular spacing and diameter of corneal fibrils, and proteoglycans are thought to play a role in collagen fibrillogenesis and matrix assembly. The keratan sulfate proteoglycans possibly function in the stroma by fitting in between and interacting with the collagen fibrils so as to maintain the fibrils at a uniform diameter and spacing. The crucial role of lumican in the regulation of collagen assembly into fibrils has been established by studies on mice homozygous for a null mutation in lumican. These animals presented skin laxity and fragility and bilateral corneal opacity (8). Furthermore, studies on chick embryo corneal development suggest that the glycosylation is very important to the corneal transparency process. Lumican with non-sulfated keratan sulfate side chains was detected on the 7th day of embryonic development but sulfated glycosaminoglycan side chains were detected only on the 15th day, when transparency starts to increase (9). These findings suggest that the sulfation of keratan sulfate in lumican may be important for the development of corneal transparency, possibly leading to organization and optimal hydration of the corneal tissue.

On the other hand, there is evidence suggesting that dermatan sulfate proteoglycans are not involved in the regulation of collagen fibril diameter, but are important for fibril- fibril spacing and lamellar cohesiveness (10). In corneal explants from chicken embryos, an increased synthesis of keratan sulfate proteoglycan and a decreased synthesis of dermatan sulfate proteoglycan coincide with the onset of tissue transparency, again suggesting a correlation between proteoglycan composition and corneal transparency (11).

Corneal wound healing following trauma or keratectomy is a major problem because the regeneration of the stromal tissue causes a subepithelial haze. Corneal scar tissue is opaque and this opacity is explained by a decreased ultrastructural order. As the regular spacing of collagen fibrils in normal adult stroma is thought to be governed by proteoglycans, the corneal haze could be related to changes in proteoglycan composition of the corneal stroma. Although many biochemical consequences of epithelial debridement by chemical, mechanical and laser treatment have been reported, controversial data were obtained by different groups concerning the corneal proteoglycan composition after epithelial injury $(12,13)$.

Although several groups have studied the glycosaminoglycan composition of the cornea, as well as the biosynthesis of proteoglycans by rabbit and chicken embryo corneal explants, only a few reports concerning human corneal glycosaminoglycans are available $(14,15)$. We have recently shown that the predominant human cornea glycosaminoglycans are dermatan sulfate and keratan sulfate (about $50 \%$ each), but these compounds were not uniformly labeled when cornea explants were incubated with ${ }^{35} \mathrm{~S}$ sulfate, suggesting lower metabolic rates for keratan sulfate (15). Furthermore, ${ }^{35} \mathrm{~S}$-heparan sulfate also appeared (12\% of labeled glycosaminoglycans) and was the main glycosaminoglycan synthesized by epithelial cells (15). Nevertheless, the proteoglycans have not been characterized.

The present paper describes the proteoglycan composition of human corneal explants and the changes that occurred when 
the corneas were submitted to mechanical epithelial debridement.

Human donor corneas that were unsuitable for transplantation were obtained from Banco de Olhos do Hospital São Paulo (São Paulo, SP, Brazil). The corneas were excised from the eyes with $1.5 \mathrm{~mm}$ scleral rims under sterile conditions, washed with $5 \mathrm{ml}$ of a 4 $\mathrm{mg} / \mathrm{ml}$ gentamicin solution in PBS and immediately placed in $12 \mathrm{ml}$ of Ham F-12 nutrient mixture supplemented with $10 \%$ FBS, 10,000 U penicillin, and $100 \mathrm{mg}$ streptomycin. These explants were cultured for various periods of time at $37^{\circ} \mathrm{C}$ in a humidified atmosphere of $2.5 \% \mathrm{CO}_{2}$. When culture time was more than three days, the nutrient medium was changed every three days.

For metabolic labeling, the corneas were trimmed free of the scleral rims and serumfree fresh Ham F-12 culture medium containing $100 \mu \mathrm{Ci} / \mathrm{ml}{ }^{35} \mathrm{~S}$-sulfate was added. The corneas were labeled with ${ }^{35} \mathrm{~S}$-sulfate for $24 \mathrm{~h}$. After ${ }^{35} \mathrm{~S}$-incorporation, the corneas were retrieved and washed twice with PBS and the proteoglycans were extracted and analyzed.

To investigate the effect of epithelial removal, before excision from the eyes the central $6.5 \mathrm{~mm}$ or the total area of the corneas was submitted to mechanical epithelial debridement. The other cornea from the same donor was maintained intact as a control. The corneas were excised and the corneal explants were submitted to the tissue culture conditions and metabolic labeling of macromolecules described above.

The corneal explants were then recovered, weighed, cut into small pieces, and suspended in $4 \mathrm{M}$ guanidine hydrochloride $(\mathrm{GuHCl})$ in $0.5 \mathrm{M}$ sodium acetate buffer, $\mathrm{pH}$ 5.8. After overnight incubation at $4^{\circ} \mathrm{C}$ with shaking, debris were removed by centrifugation and the proteoglycans were precipitated by the addition of ethanol (3 volumes) to the supernatant. Subsequently, the precipitates formed were collected by centrifugation, washed with $80 \%$ ethanol and dried. The dried material was resuspended in $100 \mu 1$ distilled water and analyzed for proteoglycans by a combination of agarose gel electrophoresis and enzymatic degradation with specific mucopolysaccharidases $(15,16)$. For the analysis of glycosaminoglycans, the proteoglycans were submitted to proteolysis with papain $(2 \mathrm{mg} / \mathrm{ml}$ in $0.06 \mathrm{M}$ phosphate-cysteine buffer, $\mathrm{pH} 6.5$, containing $20 \mathrm{mM}$ EDTA) as previously described (15). The proteoglycans and their degradation products were fixed on the gel with cetyltrimethylammonium bromide, and stained with toluidine blue. The ${ }^{35} \mathrm{~S}$-labeled compounds were visualized by exposure of the agarose gel slabs (after fixation, drying and staining) to Kodak SB-5 X-ray film for 1 to 3 days. For quantification, they were scraped off the agarose gels and counted in an Ultima Gold LSC-cocktail with a Beckman 6800 liquid scintillation spectrophotometer. Alternatively, a Packard Cyclone ${ }^{\mathrm{TM}}$ Storage Phosphor System was used to visualize and quantify the ${ }^{35}$ S-labeled compounds.

Figure 1 shows the electrophoretic migration of ${ }^{35} \mathrm{~S}$-proteoglycans and ${ }^{35} \mathrm{~S}$-glycosaminoglycans extracted from human corneal explants, stained with toluidine blue (total) and visualized by radioautography ( ${ }^{35}$ S-labeled). The figure also shows the immunoblot of these compounds with an antidecorin polyclonal antibody and with MST1, an anti-keratan sulfate monoclonal antibody (17). The proteoglycans migrated as a polydisperse band (Figure 1B, PG). Upon incubation of the proteoglycans with papain, the band corresponding to proteoglycans completely disappeared and the band(s) corresponding to free glycosaminoglycan chains could be recognized (Figure 1A). Only one band corresponding to the migration of dermatan sulfate and keratan sulfate was stained with toluidine blue (Figure 1B, GAG). In contrast, a second band migrating as heparan sulfate was identified in the radioautogram, indicating a high specific activity for this glycosaminoglycan, that was totally degraded 
by a mixture of heparitinases I and II from Flavobacterium heparinum. The band migrating as dermatan sulfate/keratan sulfate was resistant to heparitinases I and II but was partially degraded by keratanase and chondroitinase $\mathrm{B}$, indicating that it contains a mixture of keratan sulfate and dermatan sul- fate. The dermatan sulfate proteoglycan was identified as decorin by immunoblotting using a polyclonal anti-decorin antibody and the keratan sulfate proteoglycan was identified with MST1, a monoclonal antibody that recognizes keratan sulfate both as free chains and as proteoglycans. The keratan sulfate

\section{B}

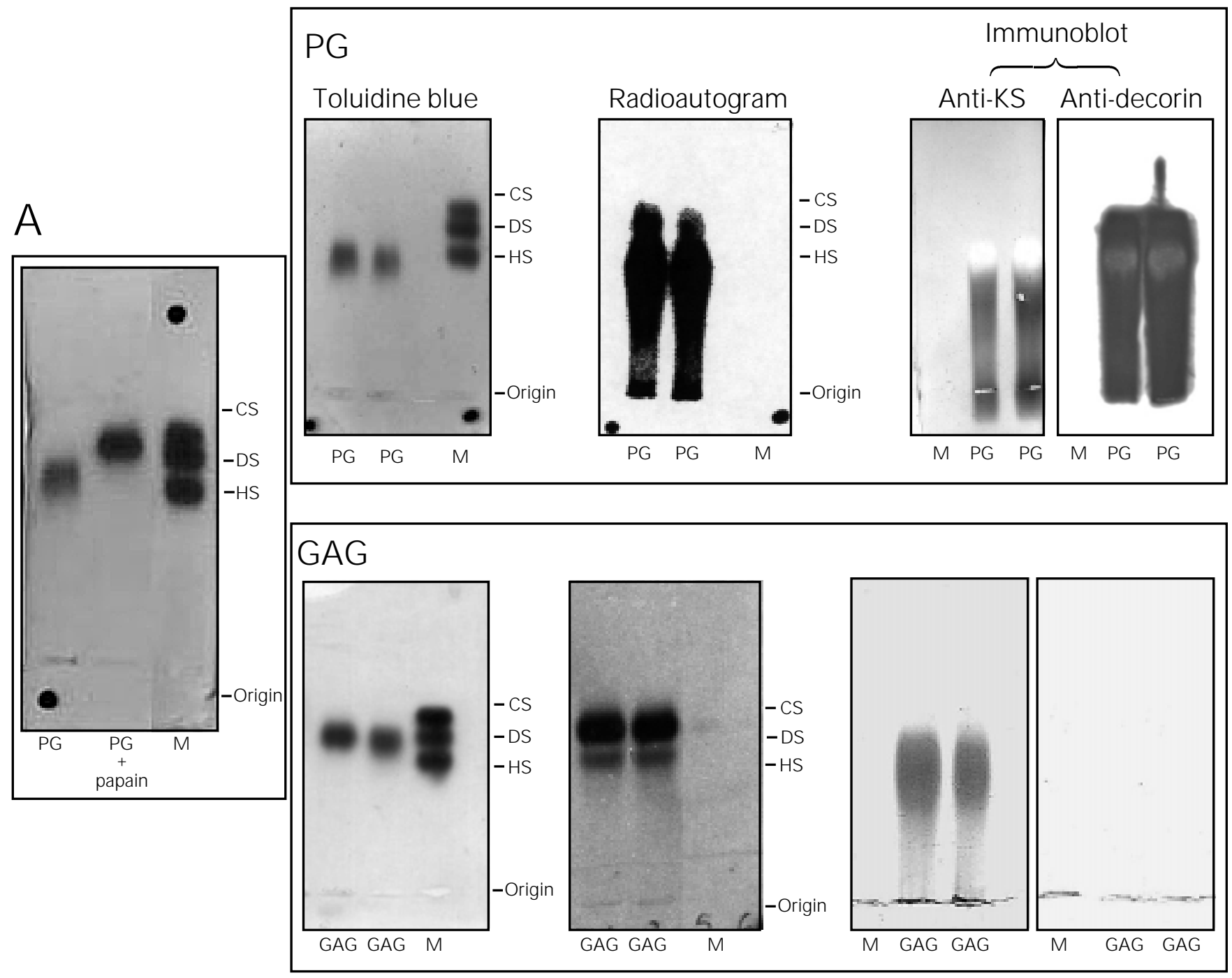

Figure 1 - Agarose gel electrophoresis of proteoglycans and glycosaminoglycans extracted from human comeas. Aliquots (5 $\mu$ l) of papain-digested proteoglycans (panel A), or intact proteoglycans (PG) and glycosaminoglycans (GAG) (panel B) extracted from human comeas were submitted to agarose gel electrophoresis in $0.05 \mathrm{M}$ 1,3-diaminopropane acetate buffer, $\mathrm{pH}$ 9.0, at $5 \mathrm{~V} / \mathrm{cm}$ for $1 \mathrm{~h}$. The proteoglycans and glycosaminoglycans were fixed in the gel by $0.1 \%$ cetyltrimethylammonium bromide and stained with toluidine blue, as previously described (15). The ${ }^{35} \mathrm{~S}-$ labeled compounds were observed by radioautography. Decorin (a dermatan sulfate proteoglycan) and keratan sulfate were also identified by immunoblotting stained with an anti-decorin polyclonal antibody and MST1 (an anti-keratan sulfate monoclonal antibody). M: Mixture of standard glycosaminoglycans containing chondroitin sulfate (CS), dermatan sulfate (DS), keratan sulfate (KS), and heparan sulfate (HS). 
staining disappeared after treatment of the glycosaminoglycans with keratanase, but not with other mucopolysaccharidases.

${ }^{35} \mathrm{~S}$-Incorporation was not uniformly distributed between dermatan sulfate and keratan sulfate (Figure 2, control). Most of the radioisotope was incorporated into derma$\tan$ sulfate, but also keratan sulfate and heparan sulfate were labeled. About $15 \%$ of the radioisotope label was found in keratan sulfate and $12 \%$ in heparan sulfate. The lower specific activity of keratan sulfate suggests a lower turnover rate for this glycosaminoglycan when compared to dermatan sulfate and heparan sulfate.

Most investigators who have studied the biosynthesis of corneal polysaccharides in adult organ culture by isotope incorporation have reported that the biosynthesis pattern changes with incubation time. A decrease in the synthesis of keratan sulfate has been reported for bovine (18) and rabbit (19) corneas, but it should be noted that the rate of synthesis does not necessarily reflect their steady-state concentrations (compare total and ${ }^{35} \mathrm{~S}$-glycosaminoglycans, Figure 2).

The epithelium of the cornea has a barrier function, impairing the diffusion of water and solutes to the stroma, as well as the penetration of microorganisms and foreign bodies. It also interacts with the tear film providing a smooth surface to the cornea, which is very important for light transmission. An active interaction between the epithelium and the stroma has been demonstrated in both normal and pathological processes, such as development, wound healing and tissue remodeling. The removal of the epithelium would modify the chemical and physical equilibrium between the epithelium and the stroma and the stromal environment would change as a consequence of the epithelial barrier damage. In the wound healing process, TGF- $\beta$, EGF, and interleukins are secreted by the epithelium and act on the stroma regulating cell proliferation, differentiation, migration, apoptosis, and extra- cellular matrix production (fibronectin and collagen) or degradation (induction of metalloproteinases). Nevertheless, the effects

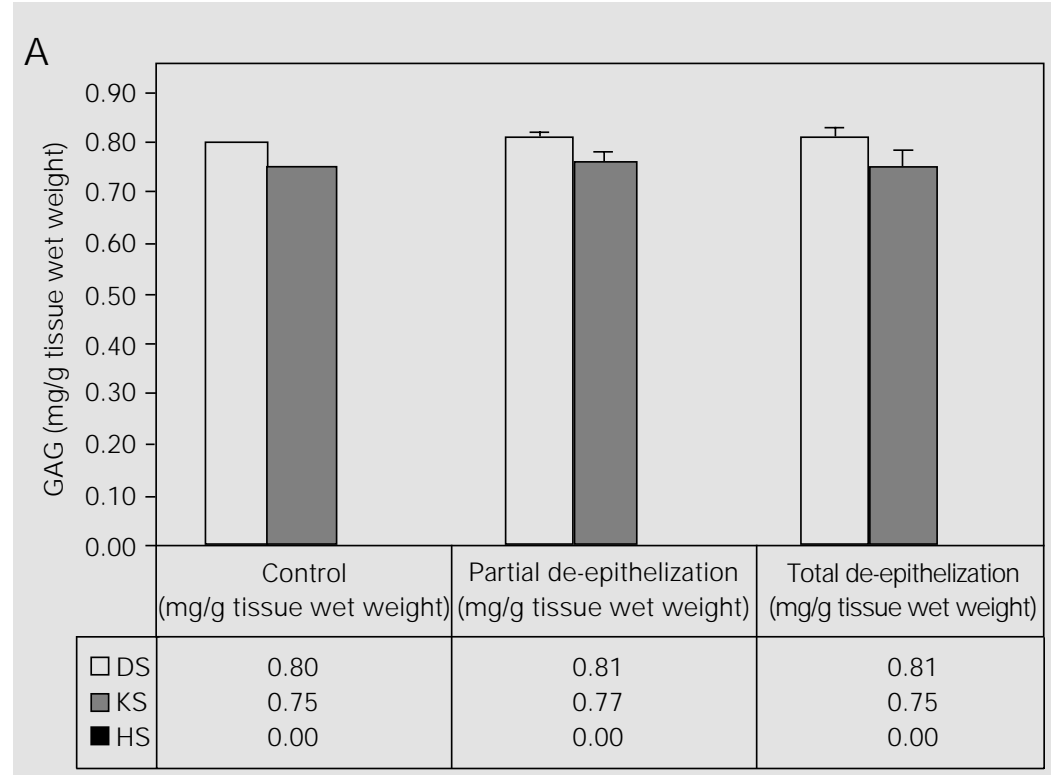

B

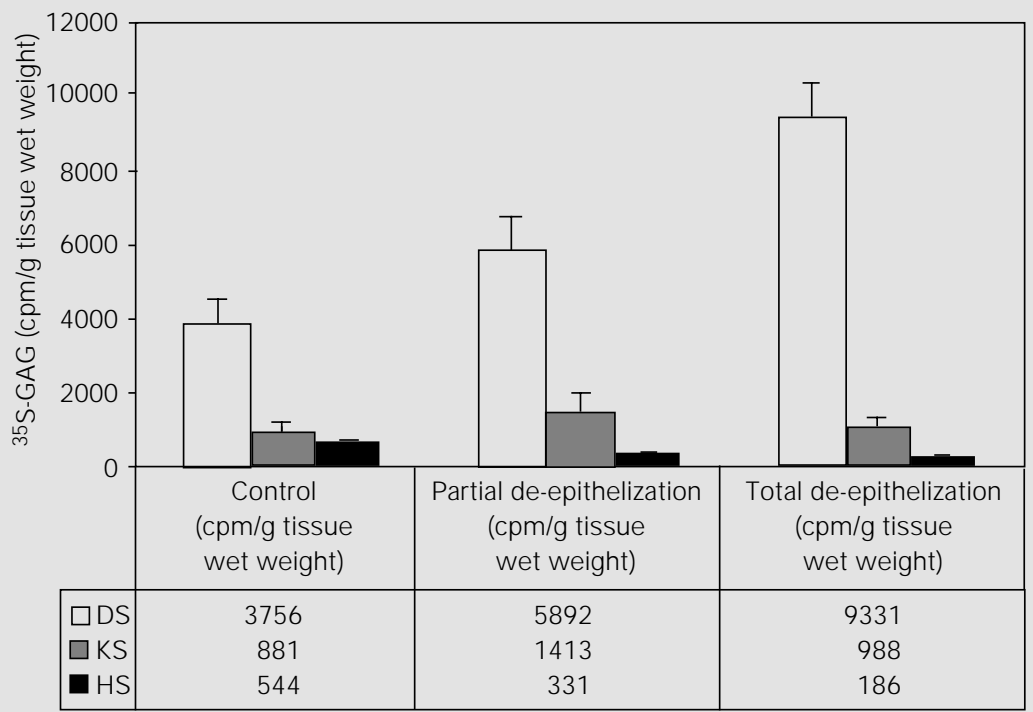

Figure 2 - Total glycosaminoglycans (A) and ${ }^{35}$ S-labeled glycosaminoglycans (B) extracted from explants of intact or epithelium-debrided human corneas. Total glycosaminoglycans were stained with toluidine blue and quantified by densitometry. The ${ }^{35}$ S-glycosaminoglycans were visualized by radioautography and scraped off the gels, and radioactivity was measured with a liquid scintillation spectrophotometer. Data are reported as means $\pm S D$ of 14 experiments. GAG, Total glycosaminoglycan; DS, dermatan sulfate; KS, keratan sulfate; HS, heparan sulfate. 
of epithelial debridement on proteoglycan synthesis are poorly understood. The events following epithelial damage might cause modifications in proteoglycan synthesis by the keratocytes.

To examine the effect of epithelial debridement on proteoglycan synthesis, the epithelial cell layer was either partially removed from a central disk $6.5 \mathrm{~mm}$ in diameter or totally removed (limbus-to-limbus). ${ }^{35} \mathrm{~S}$-Incorporation into glycosaminoglycan side chains is also shown in Figure 2. The concentration of glycosaminoglycans (expressed as $\mathrm{mg} / \mathrm{g}$ of tissue wet weight) did not change after epithelial removal (Figure 2, total glycosaminoglycans), but a drastic increase in isotope incorporation into dermatan sulfate was observed (Figure 2, ${ }^{35} \mathrm{~S}$-glycosaminoglycans). Conversely, ${ }^{35} \mathrm{~S}$-incorporation into heparan sulfate decreased. Both changes were more evident for total epithelial debridement.

It is conceivable that the epithelial layer can regulate the availability of radioactive precursors for the stromal cells. Thus, the increased radioisotope incorporation into glycosaminoglycans in corneal explants submitted to epithelial debridement may be related to increased tissue permeability to ${ }^{35} \mathrm{~S}$ sulfate. Nevertheless, the labeling of keratan sulfate was not increased, suggesting that the higher incorporation of radioisotope in der- matan sulfate is not a mere consequence of higher isotope availability but a more specific phenomenon, possibly indicating a response of stromal keratocytes to epithelial removal and/or wounding. The decrease in heparan sulfate labeling is in agreement with our previous finding that heparan sulfate is the main glycosaminoglycan synthesized by epithelial cells (at least under culture conditions) (15).

Our in vitro observations on the effects of trauma to the cornea are of interest in view of the effects of injury to the cornea observed in vivo. Lower amounts of keratan sulfate and replacement of keratan sulfate by other glycosaminoglycans have been reported in early wound healing, in corneal scars and in other pathological conditions (20). Lumican, keratocan and mimecan are transcribed and translated several times more in the cornea than in other tissues. Furthermore, a tissue-specific glycosylation of these proteins converts them to proteoglycans. Elucidation of the mechanisms by which keratocytes up-regulate expression of these genes and then provide unique glycosylation for selected sites on the proteins will greatly enhance our understanding of how this tissue obtains transparency and how transparency might be restored in pathological conditions.

\section{References}

1. Hay ED (1980). Development of the vertebrate comea. International Review of Cytology, 63: 263-322.

2. Heinegard D, Franzen A, Hedbom E \& Sommarin $Y$ (1986). Common structures of the core proteins of interstitial proteoglycans. CIBA Foundation Symposium, 124: 69-88.

3. BlochbergerTC, Vergnes J -P, HempelJ \& Hassell J R (1992). cDNA to chick lumican (corneal keratan sulfate proteoglycan) reveals homology to the small interstitial proteoglycan gene family and expression in muscle and intestine. J ournal of Biological Chemistry, 267: 347-352.

4. Funderburgh J L, Funderburgh ML, Brown
SJ , Vergnes J -P, Hassell J R, Mann MM \& Conrad GW (1993). Sequence and structural implications of a bovine corneal keratan sulfate proteoglycan core protein. Protein 37B represents bovine lumican and proteins 37A and 25 are unique. J ournal of Biological Chemistry, 268: 1187411880.

5. Chakravarti S, Stallings RL, SundarRaj N, Cornuet PK \& Hassell J R (1995). Primary structure of human lumican (keratan sulfate proteoglycan) and localization of the gene (LUM) to chromosome 12q21.3-q22. Genomics, 27: 481-488.

6. Corpus LM, Funderburgh J L, Funderburgh ML, Bottomley GS, Prakash S \&
Conrad GW (1996). Molecular cloning and tissue distribution of keratocan. Bovine comeal keratan sulfate proteoglycan 37A. J ournal of Biological Chemistry, 271: 9759-9763.

7. Li W, Vergnes J -P, Cornuet PK \& Hassell J R (1992). cDNA clone to chick corneal chondroitin/dermatan sulfate proteoglycans reveals identity to decorin. Archives of Biochemistry and Biophysics, 296: 190197.

8. Chakravarti $\mathrm{S}$, Magnuson $\mathrm{T}$, Lass $\mathrm{JH}$, J epsen KJ , LaM antia C \& Carroll H (1998). Lumican regulates collagen fibril assembly: skin fragility and corneal opacity in the absence of lumican. J ournal of Cell Biol- 
ogy, 141: 1277-1286.

9. Cornuet PK, Blochberger TC \& Hassell J R (1994). Molecular polymorphism of lumican during corneal development. Investigative Ophthalmology and Visual Science, 35: 870-877.

10. Hahn RA \& Birk DE (1992). ß-D-xyloside alters dermatan sulfate synthesis and the organization of the developing avian corneal stroma. Development, 115: 383-393.

11. Nakazawa K, Suzuki S, Wada K \& Nakazawa K (1995). Proteoglycan synthesis by comeal explants from developing embryonic chicken. Journal of Biochemistry, 117: 707-718.

12. Funderburgh J L, Stenzel-J ohnson PR \& Chandler J W (1983). Corneal glycosaminoglycan synthesis in long-term organ culture. Investigative Ophthalmology and Visual Science, 24: 208-213.
13. Nakazawa $\mathrm{K}$, Isomura $\mathrm{T} \&$ Shimoeda $\mathrm{S}$ (1995). Proteoglycan synthesis by chicken comeal explants. J ournal of Biochemistry, 117: 697-706.

14. Tai GH, Nisduszynski IA, Fullwood NJ \& Huckerby TN (1997). Human corneal keratan sulfates. J ournal of Biological Chemistry, 272: 28227-28231.

15. Soriano ES, Queiroz MC \& Michelacci YM (2000). Glycosaminoglycan synthesis by human corneal explants. Effect of epithelial debridement. Clinica Chimica Acta, 295: 41-62.

16. Cássaro CMF \& Dietrich CP (1977). Distribution of sulfated mucopolysaccharides in invertebrates. Journal of Biological Chemistry, 252: 2254-2261.

17. Alves MLM, Straus AH, Takahashi HK \& Michelacci YM (1994). Production and characterization of monoclonal antibodies to shark cartilage proteoglycan. Brazilian J ournal of Medical and Biological Research, 27: 2103-2108.

18. Balduini $C$, Brovelli $A \&$ Castellani $A A$ (1970). Biosynthesis of glycosaminoglycans in bovine cornea. The effect of uridine diphosphate xylose. Biochemical J ournal, 120: 719-723.

19. Klintworth GK \& Smith CF (1976). A comparative study of extracellular sulfated glycosaminoglycans synthesized by rabbit comeal fibroblasts in organ and confluent cultures. Laboratory Investigation, 35: 258-263.

20. Nakayasu K, Gotoh T, Ishikawa T \& Kanai A (1996). Glycosaminoglycans in subepithelial opacity after excimer laser keratectomy. Nippon Ganka Gakkai Zasshi, 100: 350-357. 